\title{
Evaluation of Chromosome Damage due to a Nitronaphtofuran Derivative: R7372 on the Plant Nigella damascena L.
}

\author{
K. Tantaoui El Araqi, J. Gilot-Delhalle and J. and \\ M. Moutschen-Dahmen \\ Université de Liège, Laboratoire de Toxicologie Génétique, \\ Sart-Tilman, B22 B-4000 Liège, Belgium
}

Accepted August 30, 1995

Numerous nitrofuran derivatives have been widely used in human and veterinarian medicine and also as food additives, due to their antiparasitic potentialities (Bryan 1978). Two nitronaphtofurans: 7-methoxy-2-nitro-naphto [2,4-b] furan [R7000] and 7-methoxy-1-methyl2 nitro-naphto [2,4-b] furan [R7372], were selected for their enhanced activities (Einhorn et al. 1983).

These compounds were found to be highly mutagenic in microorganisms (Weill-Thevenet et al. 1982, Arnaise et al. 1986) and carcinogenic in rat (Salmon et al. 1987).

R7372 was also found to be genotoxic on two strains of the yeast Schizosaccharomyces pombe (Tantaoui El Araqi 1992).

The genotoxicity strongly limits therapeutic uses of these derivatives but their enhanced mutagenic efficiency makes them valuable tools to investigate the mechanism(s) inducing chromosome damage or leading to carcinogenicity.

However, molecules of this class, with enhanced specific mutagenic and carcinogenic properties could be useful for experimental purposes (Gilot-Delhalle et al. 1994).

The chromosome breaking efficiency of the compound R7000 was previously investigated in the plant Nigella damascena in both seeds and root tips (Gilot-Delhalle et al. 1994).

The aim of the present investigation is to compare chromosome damaging activity on the compound R7372 with those of R7000 on the same plant system.

\section{Material and methods}

Nigella damascena seeds (var. Miss Jekyll blue double) "dry" ( $\left.\approx 8 \% \mathrm{H}_{2} \mathrm{O}\right)$ or presoaked ( 40 or $60 \mathrm{hr}$ ) were treated for $5 \mathrm{hr}$ at increased $\mathrm{R} 7372$ concentrations (from $31 \times 10^{-5} \mathrm{M}$ to $\left.155 \times 10^{-5} \mathrm{M}\right)$. The presoaking times correspond respectively to the $G_{0}, G_{1}$ and $S-G_{2}$ stages of the mitotic cycle. After treatment with R7372, seeds were washed and transferred in petri dishes. Root tips were immersed in a colchicine solution (Merck, $0.05 \mathrm{~g} / 100 \mathrm{ml} / 4 \mathrm{hr}$ ) $72 \mathrm{hr}$ after the onset of germination, and fixed (Carnoy, 2 hr-overnight). Slides were prepared from Feulgen squashes and scanned under double blind regimen.

Root tips ( $96 \mathrm{hr}$ germination) were treated for $4 \mathrm{hr}$ with $\mathrm{R} 7372$ at concentrations ranging from $0.97 \times 10^{-5} \mathrm{M}$ to $15.6 \times 10^{-5} \mathrm{M}$ and were washed. After intervals of $4,8,12 \mathrm{hr}$, root tips were fixed under the same conditions as described above. These times were selected for studying the sensitivity of different stages of the mitotic cycle. In Nigella, the duration of a whole mitotic cycle in root tips is about $16 \mathrm{hr}$ in our experimental conditions (Gilot-Delhalle et al. 1994).

To evaluate a possible interaction of $\mathrm{R} 7372$ with colchicine, a third set of experiments was 
performed. Root tips were treated with R7372 at increased times: 4, 8, and 12 hr corresponding to different phases of the mitotic cycle, then treated with colchicine $4 \mathrm{hr}$ before fixation.

To evaluate metaphase accumulation, the mitotic and the metaphases indices were scored on an area containing fairly constant number of meristematic cells $(\overline{\mathrm{x}} \pm \mathrm{t} 0.05 \sigma=100 \pm 9$ ). (five areas per root tip for ten root tips). All cytological observations were made with a photomicroscope Zeiss (II) (Objective $100 \times \mathrm{Ph} 3$ Neofluar).

R7372 Fig. 1 being very slightly soluble in water, an appropriate solvent had to be selected. Dimethyl sulfoxide (DMSO) did not show any toxicity at concentrations of $2.5 \% \mathrm{v} / \mathrm{v}$. R7372 solutions were prepared at room temperature $\left(22 \pm 1^{\circ} \mathrm{C}\right)$.

\section{Results}

The first observations revealed that the chromosomal lesions induced by the compound are of the same type as those induced by R7000 whatever the concentration. All lesions belong to

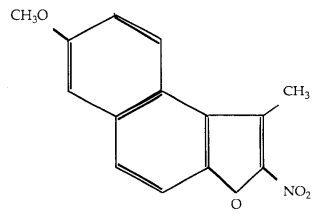

Fig. 1. Structure of R7372. the chromosome $\left(\mathrm{G}_{0}-\mathrm{G}_{1}\right)$ (before DNA synthesis) class i.e. consist in chromosome fragments or minutes (conventionally $\leq 0.5 \mu$ ) (Fig. 2a) interpreted as terminal deletions. Only few chromosome exchanges (not exceeding $1 \%$ or the total lesions) were observed. Beside chromosome fragments, a

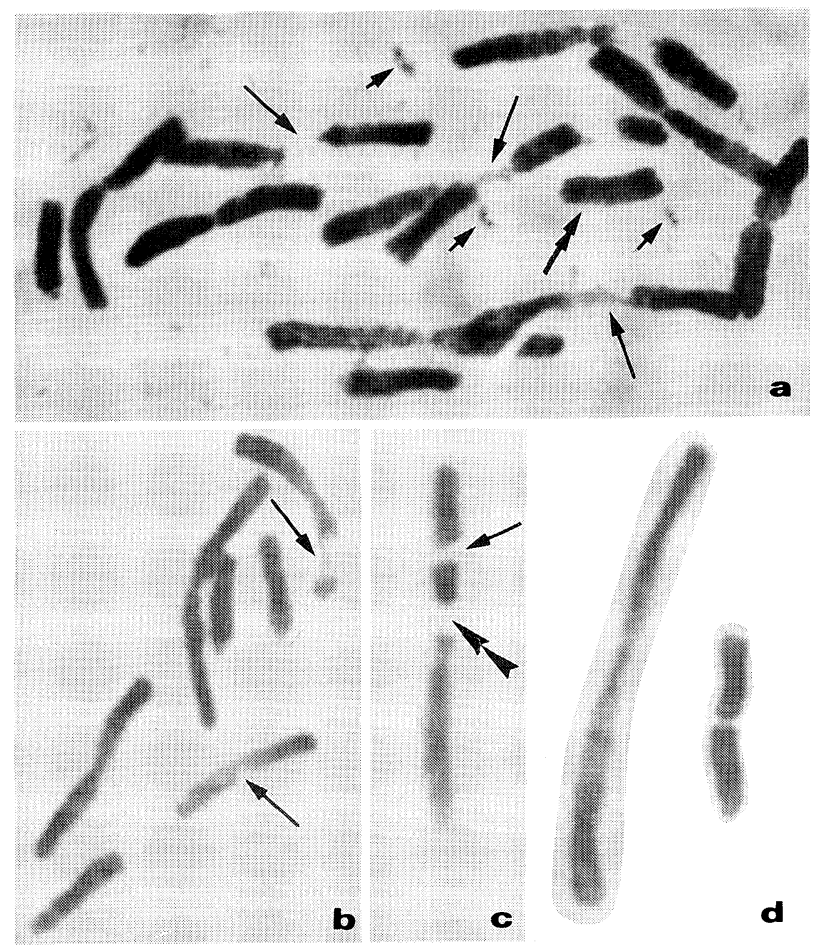

Fig. 2. a, Metaphase showing various types of lesions: apurinic regions indicated by arrows; double minutes (small arrows); acentric fragment (double arrow) $2000 \times$ GR. b, Above: chromosome uncoiling (indicated by arrow); Below: achromatic gap $\left(\mathrm{G}^{\prime}\right)$ (indicated by arrow) $1800 \times \mathrm{GR}$. c, Example of two large iso-achromatic gaps $\left(\mathrm{G}^{\prime \prime}\right) 1800 \times \mathrm{GR}$. d, Left: extreme uncoiling of a mediocentric chromosome Right: normal chromosome from a control $1800 \times \mathrm{GR}$. 
large number of chromosome gaps, either achromatic (single) (Fig. 2b) or iso-achromatic (double) which sometimes appeared as apurinic regions were noticed (Fig. 2c). Chromosomes show a general uncoiling (Fig. 2a, b, d) sometimes localized at the centromeric regions (Fig. 2a). The same types of lesions were also observed after treatments of root tips at different fixation times after the end of treatments. Lesions of the chromatid class were but exceptionally observed.

Data showing a linear relationship between doses and total damage are plotted in Fig. 3. Treatment of presoaked seeds resulted in a lesser effect than "dry" seeds and showed a decreased sensitivity of $S-G_{2}$ phases compared with $G_{0}-G_{1}$.

An additional effect of R7372 also occurring after R7000 treatment was a striking chromosome uncoiling observed after treatments of 40 and $60 \mathrm{hr}$ presoaked seeds but not "dry" seeds or root tips 4,8 , or $12 \mathrm{hr}$ after treatment (Fig. 2).

This strong uncoiling is progressively increased at doses ranging from 31.1 to $155.6 \times 10^{-5}$ $\mathrm{M}$ for seeds and 0.97 to $7.8 \times 10^{-5} \mathrm{M}$ for treated root tips.

Although it was not possible to evaluate quantitatively the chromosome uncoiling effect, it can be stated that the closer the prophase, the stronger the effect. As after R7000, this uncoiling occurs more often at or near centromeric regions (Fig. 2). The effect also counteracts the normally coiling effect of colchicine.

This is a linear relationship between dose and total damage after seed treatments. Data are plotted in Fig. 3. It can be seen that the effect is decreased when seeds are presoaked as compared with dry seeds. This suggests a decreased sensitivity of the phases $\mathrm{S}-\mathrm{G}_{2}$ as compared with the phase $G_{0}-G_{1}$. After root tip treatments, a linear relationship between dose and total effect, although at lower doses, is due to a higher sensitivity of root tips compared to seeds. These data are plotted in Fig. 4. They confirm data obtained with seeds. The longer the interval between the end of treatment and fixation, the more pronounced the effect.

$\mathrm{R} 7372$ was found to be less efficient that $\mathrm{R} 7000$ for inducing chromosome aberrations. At the concentration yielding the maximum chromosome aberrations after seed treatments $(155 \times$ $10^{-5} \mathrm{M}$ ), R7372 induced $12.9 \%$ of aberrations on "dry" seeds (Fig. 3). It was about 16 times less efficient than R7000, showing only slight variations with the mitotic stage treated (GilotDelhalle et al. 1994).

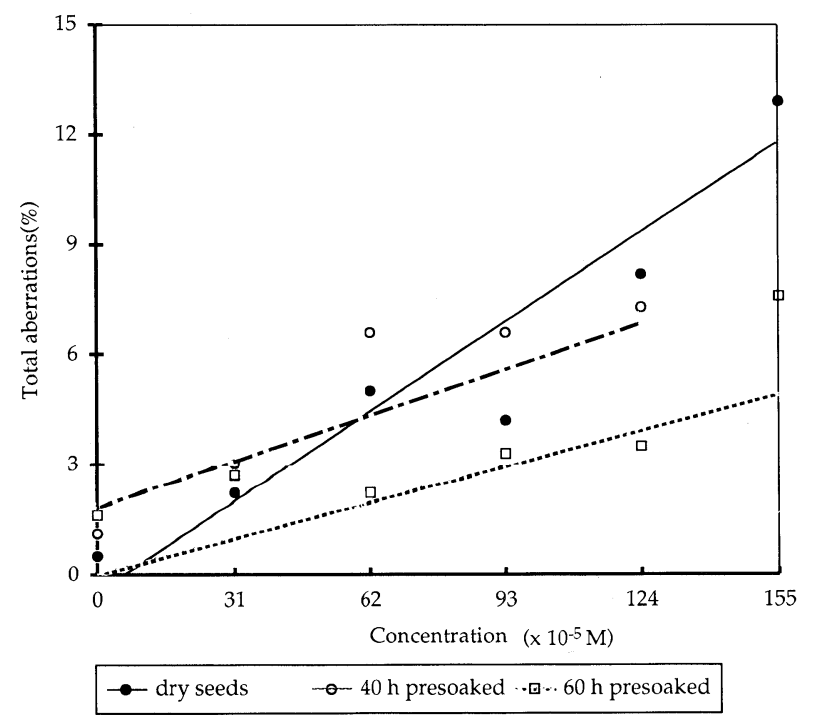

Fig. 3. Dose response-relationship after treatment of dry seeds, $40 \mathrm{hr}$ presoaked seeds and $60 \mathrm{hr}$ presoaked seeds. (\% total number of aberrations on 200 metaphases, 10 root tips in each case) 


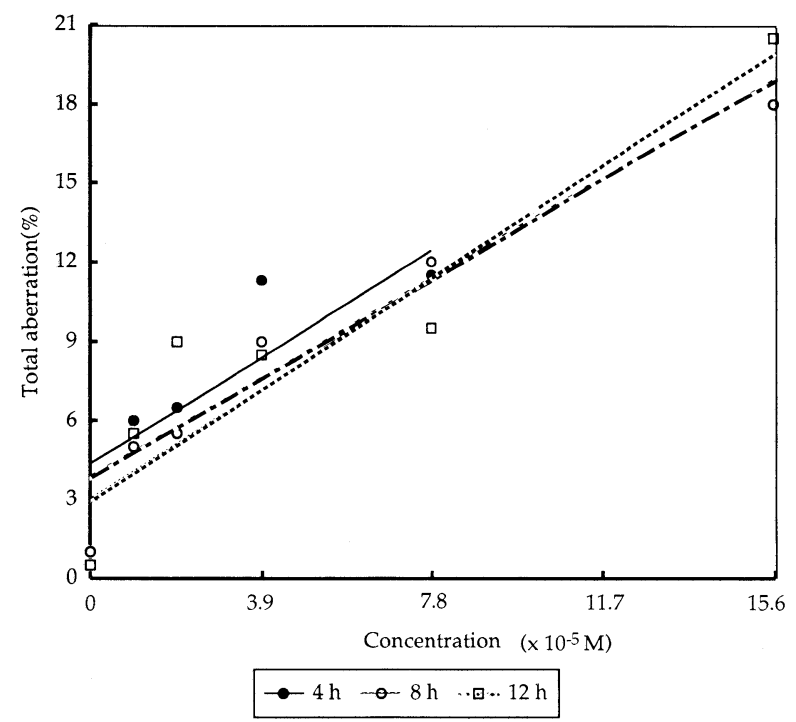

Fig. 4. Dose response-relationship after treatment of root tips for 4,8 , and $12 \mathrm{hr}$. ( $\%$ total number of aberrations on 200 metaphases, 10 root tips)

Table 1. Interaction between colchicine $(0.05 \mathrm{~g} / 100 \mathrm{ml} / 4 \mathrm{hr})$ and $\mathrm{R} 7372\left(7.8 \times 10^{-5} \mathrm{M}\right)$ for accumulating metaphases (DMSO: $2.5 \% \mathrm{v} / \mathrm{v}$ mean metaphase number) 10 root tips, 500 cells per root tip, $\overline{\mathrm{x}} \pm \mathrm{t} 0,05 \sigma$

\begin{tabular}{ccccc}
\hline $\begin{array}{c}\text { Duration of R7372 } \\
\text { treatment }\end{array}$ & Control DMSO & $\begin{array}{c}\text { Treatment DMSO }+ \\
\text { R7372 }\end{array}$ & $\begin{array}{c}\text { Control DMSO }+ \\
\text { colchicine }\end{array}$ & $\begin{array}{c}\text { Treatment R7372+ } \\
\text { colchicine }\end{array}$ \\
\hline $4 \mathrm{hr}$ & $0.5 \pm 0.5$ & $0.6 \pm 0.5$ & $2.75 \pm 0.58$ & $8.7 \pm 4.4$ \\
$8 \mathrm{hr}$ & $1.6 \pm 0.5$ & $1 \pm 0.6$ & $46.6 \pm 10.8$ & $67.7 \pm 36.1$ \\
$12 \mathrm{hr}$ & $3.1 \pm 1$ & $3.2 \pm 1.3$ & $68.1 \pm 15.3$ & $89 \pm 32.9$ \\
\hline
\end{tabular}

DMSO: Dimethylsulfoxide.

In previous experiments, some synergistic effects between $\mathrm{R} 7372$ and colchicine were suspected. A synergistic effect for metaphase accumulation was demonstrated in a third set of experiments. Data, given in Table 1 are showing that, the longer the treatment time with R7372, the stronger the metaphase accumulations (Statistical: Snedecor test for variance analysis: $F^{* *}$ for each of the four treatments).

\section{Discussion}

Qualitative effects of R7372 closely resemble those of R7000. After treatments with each compound the damage consists in chromosome fragments and microfragments interpreted as deletions and gaps interpreted as apurinic regions. This damage is of the $G_{0}-G_{1}$ type, at which stage it is quantitatively higher. There is also, after treatments of each mitotic stage by one of the two nitronaphtofurans, a drastic uncoiling of chromosomes, sometimes preferentially localised at centromeric regions, eventually leading to centromeric breaks. This uncoiling could be in some ways interpreted as a modification of the action of topo-isomerase (Gilot-Delhalle et al. 1994). The synergistic effect with colchicine for metaphases accumulation could be interpreted as an effect localised at two different sites. The growth inhibiting effect of spindle tubuline molecules due to colchicine is well known. So, the site of action of the nitronaphtofurans should be elsewhere. We suggest the kinetochore regions due to the strong localization of centromeric damage. Some mechanisms of action have been proposed for R7000, but it is 
not known till what extend they can be totally extrapolated to R7372.

Electronic disequilibrium would explain various modifications of the nucleophilic character of the the molecules which lead to mutagenic derivatives such as anionic radicals, superoxide anion diverse amines and hydroxylamine (Gilot-delhalle et al. 1994, Biaglow et al. 1977, Mc Calla 1983).

On the basis of these data, some structure-activity relationships could be expected. R7372 should induce more damage than R7000.

This is certainly not the case, neither for chromosome damage in Nigella nor reversions in Schizosaccharomyces pombe (Tantaoui El Araqi 1992). On the assumption of the mutagenic efficiency of some derivatives, differences of activity could be due to differential metabolism in various organisms or even tissues, e.g. the accumulation of hydroxylamines could explain most of our experiments.

\section{Summary}

Chromosome damage induced by R7372 was investigated in Nigella damascena seeds (doses ranging from 31 to $155 \times 10^{-5} \mathrm{M}$ ) and root tips (doses ranging from 0.97 to $15.6 \times 10^{-5}$ M). Chromosome damage consisted in fragments, microfragments and gaps. There was also a chromosome uncoiling effects of R7372 and a synergistic effects with colchicine for metaphase accumulation. A linear relationship between dose and total effect was established for both seeds and root tips. The damage and the sensitivity of various phases of the mitotic cycle could be compared with the effect of another nitronaphtofuran (R7000).

Key word : Cytological observations, Nitronaphtofuran, Nigella damascena.

\section{Acknowledgments}

The authors are thankful to Dr J.-P. Buisson from the Institut Curie (Paris) for generous gifts of R7372.

\section{References}

Arnaise S., Boeuf H., Buisson J.-P., Cantat N., Demerseman P., Einhorn J., Lamotte G., Lemelin M., Hsie A. W., Royer R., Kelly F. and Hofnung M. 1986. Genotoxic activities of 2-nitronaphtofurans and related molecules. Mutagenesis 1: 217-229.

Biaglow S. E., Jacobson B. E. and Nygard O. F. 1977. Metabolic reduction of 4-nitroquinolin N-oxide and other radical producing drugs to oxygen reactive intermediates. Cancer Res. 37: 3306-3313.

Bryan G. T. 1978. Nitrofurans: chemistry, metabolism, mutagenesis and carcinogenesis. Carcinogenesis 4: 1-11, Nitrofurans, Raven Press, N. Y.

Einhorn J., Demerseman P., Royer R. and Cavier R. 1983. Recherche sur les dérivés nitrés d'intérêt biologique. Eur. J. Med. Chim. 18: 175-180.

Gilot-Delhalle J., Moutschen J. and Moutschen-Dahmen M. 1994. Clastogenic effects of methoxy-7-nitro-2-naphto $(2,1 b)$ furan (R7000) in Nigella damascena. Environ. Exp. Bot. 34: 451-460.

Marfey P. and Robinson E. 1981. The genetic toxicology of hydroxylamine. Mutat. Res. 86: 155-191.

Mc Calla D. R. 1983. Mutagenic of nitro-naphtofuran derivatives. Envir. Mut. 4: 745-765.

Moreno S. N. S. and Docampo R. 1985. Mechanism of toxicity of nitro-compounds used in the therapy of trichomonasis. Envir. Health Res. 64: 199-208.

Salmon R. J., Buisson J. P., Demerseman P., Einhorn J., Ausseppe L., Zafrani B., and Royer R. 1987. Comparison of the carcinogenic effects of two 2-nitro-naphtofurans injected subcutaneously in rats. Cancer Letters 35: 5964.

Tantaoui El Araqi K. 1992. Etude des effects mutagènes chez Schizosaccharomyces pombe Lindner et des effets clastogènes chez Nigella damascena L. d'un nitro-naphtofuranne, le le 7-methoxy-1-methyl-2 nitonaphto- 
[2,1b]furanne (R7372) Bull. Soc. Roy. Sci. Lg. 61: 407-421.

Weill-Thévenet N., Buisson J.-P., Royer R. and Hofnung M. 1982. Genetic toxicology studies with 2-nitrobenzofurans and 2-nitronaphtofurans. Mutat. Res. 104: 1-8. 